\title{
ANALYSIS OF FORMALDEHYDE COMPOSITION EFFECT ON THE MORPHOLOGY OF POLY (UREA-FORMALDEHYDE) MICROCAPSULE CONTAINING LINSEED OIL
}

\author{
BAYU PRASETYA* \\ Department of Materials and Metallurgical Engineering \\ Institut Teknologi Sepuluh Nopember. \\ Jalan Teknik Kimia, Keputih, 60111, Surabaya, East Java, Indonesia \\ materials.bayu@gmail.com \\ AGUNG PURNIAWAN \\ Department of Materials and Metallurgical Engineering \\ Institut Teknologi Sepuluh Nopember, \\ Jalan Teknik Kimia, Keputih, 60111, Surabava, East Java, Indonesia \\ agung pur@mat-eng.its.ac.id \\ HOSTA ARDHYANANTA \\ Department of Materials and Metallurgical Engineering \\ Institut Teknologi Sepuluh Nopember, \\ Sukolilo, 60111, Surabaya, East Java, Indonesia \\ hosta@mat-eng.its.ac.id \\ * Corresponding author
}

\begin{abstract}
Microcapsules technology for smart materials application were developed recently years. One of the smart materials was selfhealing coating. The microcapsules containing healing agent are dispersed into a polymeric matrix to form self-healing composite. Additionally, the microcapsules might be incorporated into paint for coating application. The main idea of self-healing coating is to design smart self-healing systems with inherent ability of self-repair. Many studies that have been done proposed several healing stimulus mechanisms. Commonly the capsules shell composed from organic polymers, such as urea-formaldehyde, polyurethane, and melamine formaldehyde. In this research, poly (urea-formaldehyde) (PUF) microcapsules containing linseed oil were synthesized by in-situ polymerization method. The obtained microcapsules were analyzed based on parameter of formaldehyde to urea mass ratio $(2,3,4$, and 5$)$. The result show that the increase of formaldehyde to urea ratio increased the microcapsules shell thickness.
\end{abstract}

Keywords: in-situ polymerization, microcapsules, poly (urea-formaldehyde), self-healing coating

\section{Introduction}

Microcapsules technology for smart materials application were developed recently years. One of the smart materials was self-healing coating. The microcapsules containing healing agent are dispersed into a polymeric matrix to form self-healing composite. Additionally, the microcapsules might be incorporated into paint for coating application. The main idea of self-healing coating is to design smart self-healing systems with inherent ability of self-repair. In order to reduce the impact or prevent corrosion in the gap that has already formed in the coating, it is necessary to have a type of coating that has two functions, namely self-cleaning and self-healing as well. The role of self-cleaning is to prevent water from being absorbed through the metal surface and selfhealing is needed so that the coating is able to repair the damage itself (Makhlouf, 2014).

There are two types of self-healing mechanisms, namely autonomous and non-autonomous. Autonomous mechanism is a mechanism to repair coating automatically without being influenced by the external environment. An autonomous self-healing coating contains a healing agent that is capable of polymerizing or using a corrosion inhibitor. While the mechanism of non-autonomous self-healing coating, the repair process 
requires external factors such as heat or light as a stimulus for the formation of chemical bonds (Zhang et. al., 2018)

Many studies have proposed several healing stimulus mechanisms. Commonly the capsules shell composed from organic polymers, such as urea-formaldehyde, polyurethane, and melamine formaldehyde. (Katoueizadeh et. al., 2019). Urea-formaldehyde polymers are composed of urea and formaldehyde molecules. This is basically similar to phenol-formaldehyde. Urea-formaldehyde can be used as a binding material for weather resistant plywood, dyes, plastic furniture, or coatings on metal furniture (Higgins, 2006). Formaldehyde is the smallest aldehyde consisting of two hydrogen atoms and an oxygen atom that binds to a carbon atom in the middle. Formaldehyde has the chemical formula $\mathrm{CH} 2 \mathrm{O}$ with the chemical structure shown in Figure 2.12. Formaldehyde has been widely used in various fields. $37 \%$ solution, for example, is used in preserving biological samples because of its ability to cross-link protein (He, 2017).

Linseed oil is composed of glycerol ester groups (C3 alcohol with 3 hydroxyl groups) and 5 fatty acids, 2 of which are saturated, namely $\mathrm{C} 16$ palmitic and $\mathrm{C} 18$ stearic, and 3 unsaturated namely oleic, linoleic, and linolenic. Figures 2.15 and 2.16 show the molecular chain structure of linolenic acid and flaxseed oil. Flaxseed oil contains $60 \%$ linolenic acid, which is far greater compared to other types of oils. The content of linolenic acid influences the speed of drying when exposed to air. The greater the content of linolenic acid, the faster it will dry (Dlugogorski et. al., 2012).

Some studies have been synthesized microcapsules containing linseed oil through in-situ polymerization methods with poly (urea-formaldehyde) (PUF) as shell material. However, the obtained microcapsules still have poor mechanical properties. The size and concentration of the microcapsules in the resin also affect the corrosion resistant performance of self-healing coating (Behzadnazab et. al., 2017).

In this research, poly (urea-formaldehyde) (PUF) microcapsules containing linseed oil were synthesized by in-situ polymerization method. The obtained microcapsules were analyzed based on parameter of formaldehyde to urea mass ratio $(2,3,4$, and 5$)$.

\section{Experimental}

\subsection{Materials}

Urea (Smart Lab Indonesia), polyvinyl alcohol (PVA), formaldehyde aqueous solution 37\%, ammonium chloride, resorcinol, $\mathrm{HCl}$ (Merck Chemical Germany), linseed oil (Swisloyd Belgium)

\subsection{Syntesis of PUF microcapsules}

Linseed oil encapsulated PUF microcapsules were prepared by in-situ polymerization method. PVA aqueous solution ( $5 \mathrm{wt} \%) 7.5 \mathrm{ml}, 130 \mathrm{ml}$ distiled water, $5 \mathrm{~g}$ of urea, $0.5 \mathrm{~g}$ of ammonium chloride, and $0.5 \mathrm{~g}$ of resorcinol were mixed using mechanical stirrer (purchased from IKA) at $600 \mathrm{rpm}$ for 15 minutes. Then, the $\mathrm{pH}$ value of solution was adjusted to 3 by adding $1 \mathrm{wt} \% \mathrm{HCl}$ aqueous solution. While stirring at $800 \mathrm{rpm}, 20 \mathrm{ml}$ linseed oil was added dropwise into solution, followed by the addition of formaldehyde with mass variation $10,15,20$, and $25 \mathrm{~g}$. The emulsion was heated to $55{ }^{\circ} \mathrm{C}$ and kept for $4 \mathrm{~h}$. The microcapsules were collected by vacuum filtration and washed with distiled water. The washed microcapsules then dried for $24 \mathrm{~h}$ at room temperature. The scheme of in-situ polymerization method is illustrated by Fig.1.

\subsection{Characterization}

A investigation of microcapsules structure was accomplished by an optical microscope (Olympus CX31). Then the microcapsules diameter was measured by image rasher software. A more detailed examination of microcapsule surface morphology was carried out by scanning electron microscope (SEM) (FESEM Hitachi 1000). Fourier transform infrared spectrometer (FTIR) (Nicolet iS10 FITR Spectrometer) was used to identify the chemical structure of microcapsules. The synthesis conditions for formation of PUF microcapsules are shown in Table 1. 
Table 1. Synthesis condition for formation of PUF microcapsules and their identification codes

\begin{tabular}{ccccc}
\hline \hline & \multicolumn{4}{c}{ Variable } \\
\cline { 2 - 5 } Sample code & $\begin{array}{c}\text { Linseed oil } \\
(\mathrm{ml})\end{array}$ & Urea $(\mathrm{g})$ & $\begin{array}{c}\text { Formaldehyde } \\
(\mathrm{g})\end{array}$ & F/U \\
\hline MF2 & 20 & 5 & 10 & 2 \\
MF3 & 20 & 5 & 15 & 3 \\
MF4 & 20 & 5 & 20 & 4 \\
MF5 & 20 & 5 & 25 & 5 \\
\hline
\end{tabular}

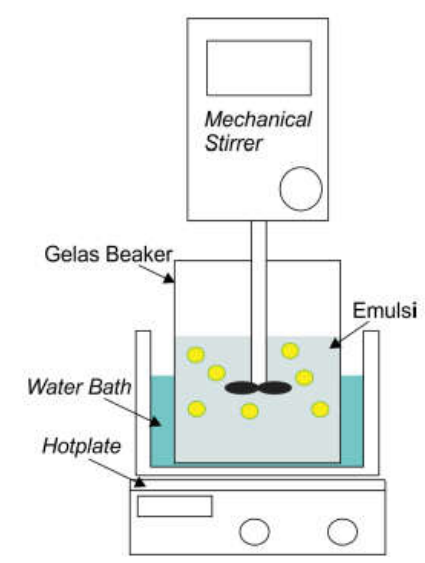

Fig. 1. Illustration of in-situ polymerization process

\section{Results and Discussion}

\subsection{Morphology of PUF microcapsules}

The synthesis process was carried out to obtain PUF microcapsules containing linseed oil as a self-healing coating material. The synthesis method uses in-situ polymerization, referring to the potential for success carried out by several literary sources. Some parameters and variations are used based on previous studies with several different variations to obtain optimum results. The formation of microcapsules occured via condensation proccess between urea and formaldehyde. PVA solution acted as a surfactant that reduced surface tension. Linseed oil was encapsulated by crosslinked urea-formaldehyde which formed microcapsules. After urea was mixed in the solution, resorcinol as a cross-linking agent and ammonium chloride as an initiator were also added. The $\mathrm{pH}$ value was set at value 3 to provide an acidic atmosphere and initiated the condensation process. Linseed oil as a self-healing agent was added dropwise into the solution while stirring at high rotation speed to form small droplets. Linseed oil was mixed by dropping produces a round droplet which then becomes a suspension in the mixture. Formation of microcapsules began after the addition of formaldehyde while still stirring until suspension formed. After the stirring process was stopped, a suspension consisting of three parts was formed. The upper layer is microcapsules and the rest of linseed oil, the middle layer is water, and the bottom layer is the residual of PUF that was destroyed and did not form microcapsules. 
Prasetya et. al.

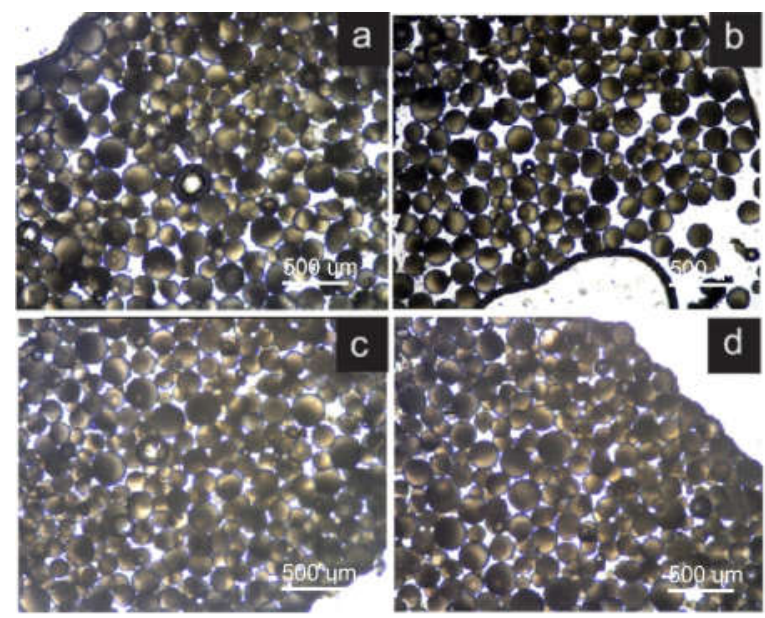

Fig. 2 Optical micrograph on microcapsules

Fig.2 shows the optical micrograph on microcapsules. These observations provided information that microcapsules have formed in spherical form. A number of microcapsules were broken and had asymmetrical shape which indicated that the microcapsule shell had poor mechanical properties. The good microcapsules for self-healing coating applications had proper mechanical properties so that they were not easy to break by physical impact. The average diameter of the microcapsules was measured by rasher image software of 50 microcapsules. Fig.3 shows the average diameter of microcapsules. The average diameter of microcapsules MF2, MF3, MF4, and MF5 sequently were 148.97, 123.11, 116.59, and $114.40 \mu \mathrm{m}$. The differences in the diameter of the microcapsules were very small. In other words the addition of formaldehyde composition gave unsignificant effect on microcapsules size.

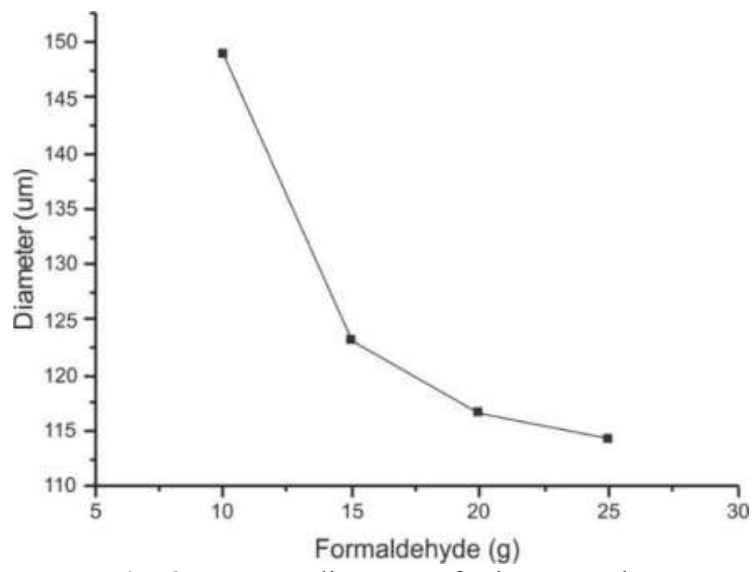

Fig. 3 Average diameter of microcapsules 
Prasetya et. al.

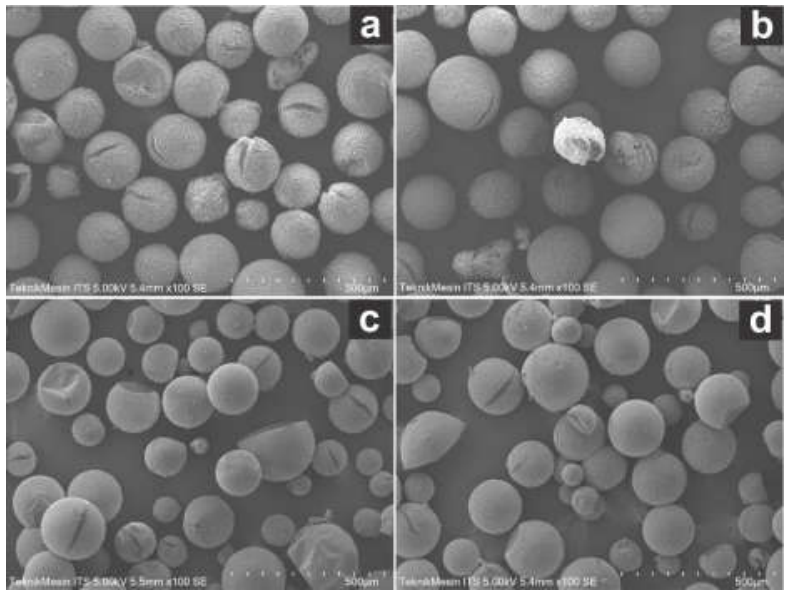

Fig. 4. SEM images of microcapsules

SEM analysis was also carried out to obtain better quality of visual micrograph. Fig.4 shows SEM images of microcapsules. Microcapsules appeared in spherical shape and large size distribution. Some microcapsules also appeared got ruptured and deflated. Shell surface of MF2 and MF3 microcapsules which have less formaldehyde composition appeared to be rougher when compared to MF4 and MF5 microcapsules which containing more formaldehyde composition.

\subsection{FTIR Spectra}

Fig.5 shows the FTIR spectra of MF2 which has an asymmetrical stretching C-H bond with strong intensity at $2923.23 \mathrm{~cm}^{-1}$, symmetrical stretching $\mathrm{C}-\mathrm{H}$ bond at the peak $2853.15 \mathrm{~cm}^{-1}$. There are esters and carboxylic acids with a type of $\mathrm{C}=\mathrm{O}$ stretching bond at the peak $1735.78 \mathrm{~cm}^{-1}$. Some of these bond types have similarities with FTIR spectra of linseed oil. The $\mathrm{CH} 2$ bond at the peak $1459.31 \mathrm{~cm}^{-1}$, the $\mathrm{C}-\mathrm{O}$ stretching bond at $1158.65 \mathrm{~cm}^{-1}$, and the asymmetrical stretching $\mathrm{C}-\mathrm{O}$ bond at the peak $1099.65 \mathrm{~cm}^{-1}$. At the peak of $3327.77 \mathrm{~cm}^{-1}$ there is a medium intensity spectra which is a type of $\mathrm{N}-\mathrm{H}$ stretching bond that has similarities to the FTIR spectra of PUF.

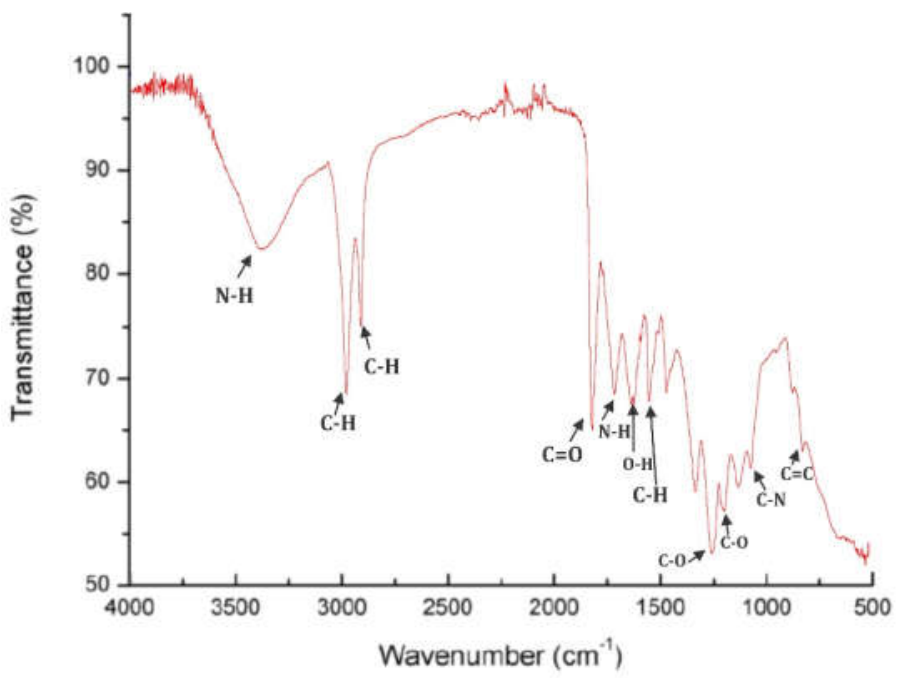

Fig. 5 FTIR spectra of MF2 
Prasetya et. al.

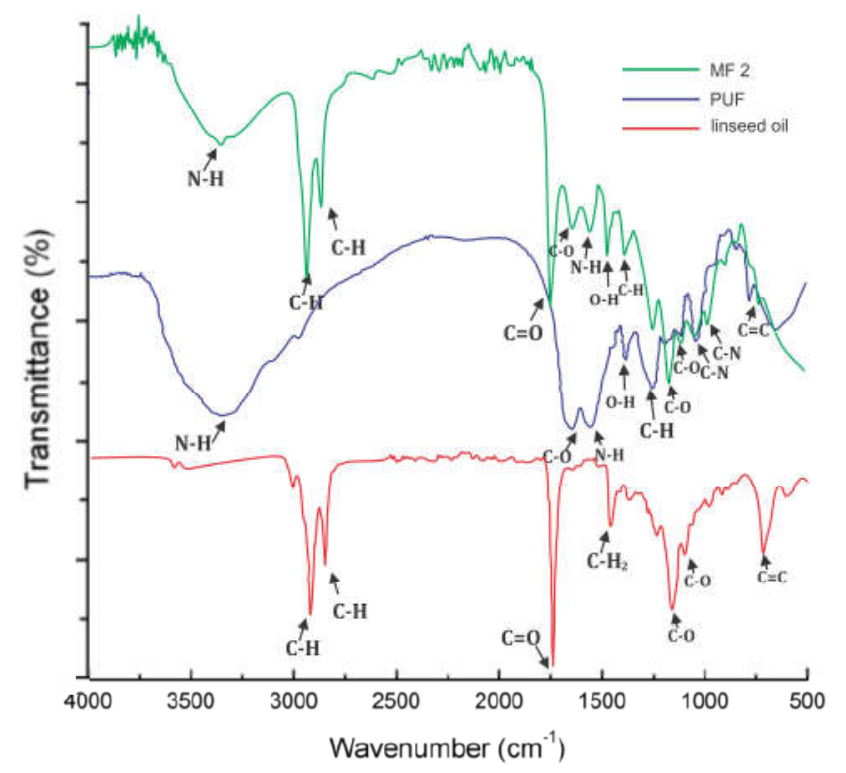

Fig. 5 FTIR spectra of linssed oil, PUF, and MF2

The FTIR transmittance spectra of linseed oil, PUF2, and MF2 are shown in Fig.5. The figure shows that the microcapsules have the same functional groups as PUF and linseed oil. The functional group of the microcapsule spectra is a combination of the functional group owned by PUF and linseed oil. This proves that the encapsulation process has succeeded in forming a microcapsule with PUF shell and containing linseed oil.

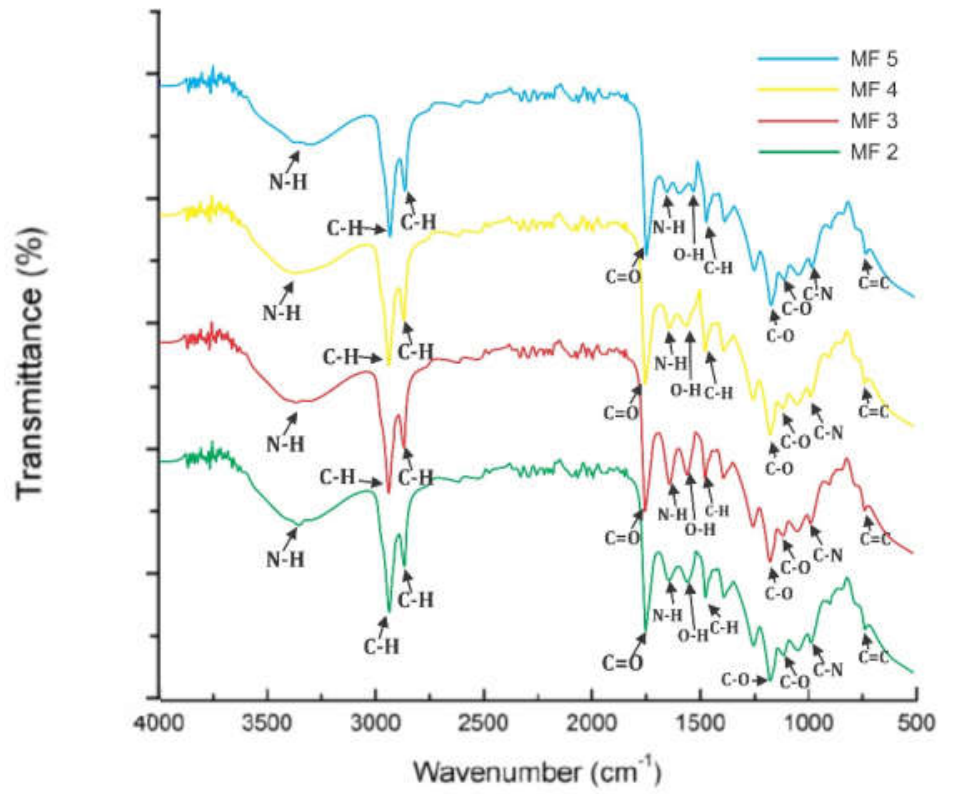

Fig. 6 FTIR spectra of linssed oil, PUF, and MF2

Fig.6 shows the FTIR spectra of all microcapsule samples. Each microcapsule sample spectra has the same functional group. Transmittance values have increased for samples sequentially MF2, MF3, MF4 and MF5. The increase in transmittance value indicates that for each addition of the formaldehyde composition, a larger 
polymer molecule was formed and resulted more microcapsules. The size of the polymer molecules formed also correlated with the thickness of the shell from the microcapsules. Microcapsules with thin shell will be vulnerable to damage. In the process of mixing the microcapsule coating can break. When the microcapsules break, the linseed oil will come out and dry out prematurely. When there is damage to the coating, the linseed oil which acts to close the gap is only left a little so that the damaged area would not be covered properly.

\section{Conclusions}

The PUF microcapsules containing linseed oil have been successfully synthesized by in-situ polymerization. The microcapsules had spherical shape with average diameters 114.40 - $148.97 \mu \mathrm{m}$. High composition of formaldehyde increased the microcapsules shell thickness. However, the formaldehyde composition gave unsignificant effect to microcapsules diameter.

\section{Acknowledgments}

The author would like to thank all those who have provided support in the work of this research.

\section{References}

Behzadnazab, M., Mirabedini S.M., Esfandeh M., and Farnood R. (2017) 'Progress in Organic Coatings Evaluation of corrosion performance of a self-healing epoxy-based coating containing linseed oil-filled microcapsules via electrochemical impedance spectroscopy', Progress in Organic Coatings. Elsevier B.V., 105, pp. 212-224. doi: 10.1016/j.porgcoat.2017.01.006

Dlugogorski, B. Z., Kennedy, E. M. and Mackie, J. C. (2012) 'Low temperature oxidation of linseed oil : a review', pp. 1-36

He, Rongqiao. (2017) 'Formaldehyde and Cognition'. Formaldehyde from Environment. Springer. (January 2015).

Higgins, Raymond A. (2006) Materials for Engineers and Technicians. Fourth Edition. Elsevier Ltd.

Katoueizadeh, Elham., Zebarjad S., and Janghorban K. (2018) 'Investigating the effect of synthesis conditions on the formation of urea - formaldehyde microcapsules', Integrative Medicine Research. Korea Institute of Oriental Medicine, 8(1), pp. 541-552. doi: 10.1016/j.jmrt.2018.04.013.

Makhlouf, A. S. H. (2014) Handbook of Smart Coatings for Materials Protection. Cambridge: Woodhead Publishing Limited

Zhang, F., Pengfei Ju, Mengqiu Pan, Dawei Zhang, Yao Huang, Guoliang Li, and Xiaogang Li. (2018) 'Selfhealing mechanisms in smart protective coatings : A review', Corrosion Science. Elsevier, 144(December 2017), pp. 74-88. doi: 10.1016/j.corsci.2018.08.005. 